Research Paper

\title{
A novel immune-related long non-coding RNAs risk model for prognosis assessment of lung adenocarcinoma
}

\author{
Songmei Lu ${ }^{1}$, Nan Shan ${ }^{2}$, Xingyue Chen ${ }^{1}$, Fangliang Peng ${ }^{2}$, Yiming Wang ${ }^{1}$, Hao Long ${ }^{3}$ \\ ${ }^{1}$ Department of Medical Oncology, Chongqing University Cancer Hospital, Chongqing, China \\ ${ }^{2}$ Department of Gynaecology and Obstetrics, The First Affiliated Hospital of Chongqing Medical University, \\ Chongqing, China \\ ${ }^{3}$ Department of Biological Immunotherapy, Chongqing University Cancer Hospital, Chongqing, China
}

Correspondence to: Yiming Wang, Hao Long; email: wangyimingcqch@163.com, https://orcid.org/0000-0002-6969-1976; lyq9167@163.com, https://orcid.org/0000-0002-3008-655X

Keywords: LUAC, long non-coding RNAs, immune-related risk score model, OS

Received: August 6, $2021 \quad$ Accepted: November 23, $2021 \quad$ Published: December 14, 2021

Copyright: (C) 2021 Lu et al. This is an open access article distributed under the terms of the Creative Commons Attribution License (CC BY 3.0), which permits unrestricted use, distribution, and reproduction in any medium, provided the original author and source are credited.

\section{ABSTRACT}

Background: The abundant immune-related long non-coding RNA (IRLNRs) in immune cells and immune microenvironment have the potential to forecast prognosis and evaluate the effect of immunotherapy. IRLNRs analysis will provide a new perspective for LUAC research.

Methods: We calculated the immune score of each sample according to the expression levels of immunerelated genes (IRGs) and screened the survival-related IRLNRs (sIRLNRs) by Cox regression analysis. The expression levels of AC068338.3 and AL691432.2 in tissues and cell lines were confirmed by RT-qPCR.

Results: 36 IRLNRs were selected by Pearson correlation analysis. Ten sIRLNRs were significantly correlated with the clinical outcomes of LUAC patients. Five sIRLNRs were identified by multivariate COX regression analysis to establish the immune-related risk score model (IRRS). The overall survival (OS) in the high-risk group was shorter than that in the low-risk group. IRRS could be an independent prognostic factor with significant survival correlation. The distributions of immune gene concentrations were different between high-risk group and lowrisk group. Furthermore, we further verified that the expression levels of AC068338.3 and AL691432.2 in different LUAC cell lines and tumor tissues were lower than that in Human bronchial epithelial cell (HBE) and adjacent tissues respectively. The lower expression levels of AC068338.3 and AL691432.2 were detected with the more advance T-stages.

Conclusions: Our results highlighted some sIRLNRs with significant clinical correlations and demonstrated their monitored and prognostic values for LUAC patients. The results of this study may provide a new perspective for immunological research and immunotherapy strategies.

\section{INTRODUCTION}

With higher mortality rate than breast, colorectal and cervical cancers combined, lung cancer (LCa) becomes a type of common malignant tumor and causes a large proportion of cancer deaths worldwide (accounting for $18.4 \%$ of all cancer deaths) [1,2]. The mainly histological subtypes of $\mathrm{LCa}$ are small cell lung cancer (SCLC, accounting for 20\%) and nonsmall cell lung cancer (NSCLC, accounting for 80\%)
[3]. NSCLC are further classified into adenocarcinoma, squamous cell carcinoma and large cell subtype [3, 4]. With the higher proportion than squamous cell carcinoma and large cell subtype, lung adenocarcinoma (LUAC) which accounting for appropriated $60 \%$ of NSCLC is the most common subtype of NSCLC [5-7].

Although radical resection brought hope to patients with $\mathrm{LCa}$, the recurrent rate within 5 years, reaching near 
$50 \%$ [8]. Therefore, increasing number of studies have been exploring more treatment strategies including chemotherapy, radiotherapy, biological targeting and immunotherapy [9]. In recent years, a growing body of immunotherapeutic options, such as PD-1 and PD-L1 blockers, have been approved for LCa, and achieved satisfied outcomes [10, 11]. Additionally, certain studies have revealed the expression levels of immunerelated markers such as PD-1 and PD-L1, are remarkably correlated to the effects of immunotherapy [12]. Therefore, the further discoveries of biomarkers of immunotherapeutic effects and prognosis are anticipated.

As the important regulatory factors of gene expression, tumor microenvironment (TME) is closely associated with the tumorigenesis, progression and prognosis [13, 14]. For the past few years, increasing numbers of immune-related genes (IRGs) were identified to help predict prognosis of various cancers [15-17], and some biomarkers in IME have also been documented to assess the immunotherapeutic response $[18,19]$. Nevertheless, in the aspect of markers of IRGs, there are still some deficiencies, especially for the prediction of the prognosis of LUAC.

Accumulating evidence has ascertained that long noncoding RNAs (LncRNAs) participate in regulating the immune response and prognosis of tumor [20, 21], and certain immune-related LncRNAs (IRLNRs) are implicated in prognosis evaluation of patients with tumors [22, 23]. Overexpression of IncRNA-MALAT1 has been explored to associate with the high recurrence rate of hepatocellular carcinoma after liver transplantation [24]. LncRNA-DANCR attenuates SOCS3 transcription and promotes breast cancer progression [25]. TMPO-AS1 was demonstrated to contribute to the tumorigenesis and progression of bladder cancer by regulating mir-98a-5p [26]. Besides, Wei et al. illuminated that TMPO-AS1 promotes the proliferation of LUAC cells via regulating miR-326/SOX12 axis [27]. AL606489.1 was also verified to induce ferroptosis and autophagy in lung cancer [28, 29]. Although increasing studies have clarified the roles of IRLNRs in various tumors, their predicting potential for patients with LUAC remain unclear.

Therefore, we sought to explore the clinical significance of IME and IRLNRs on forecasting the prognosis of LUAC. The study establishes an accurate and appropriate model for prognosis prediction of LUAC patients and contributes to reveal the underlying mechanisms of the forecasting effects of IRLNRs in future researches. More importantly, the results will offer a guiding basis for clinicians.

\section{MATERIALS AND METHODS}

\section{Clinical samples and cell lines}

Tumor and normal adjacent tissues of LUAC patients were obtained from 374 patients admitted to Chongqing University Cancer Hospital, of which 106 T1 stage patients, 192 T2 stage patients, 60 T3 stage patients and $16 \mathrm{~T} 4$ stage patients. Human bronchial epithelial cell (HBE) and LUAC cell lines (A549, HCC827, NCI-H1299, NCI-H2228 and NCI-H1975) were obtained from the American Type Culture Collection (ATCC, Virginia, USA). DMEM and 1640 medium, adding $10 \%$ fetal bovine serum, $100 \mu / \mathrm{ml}$ penicillin and $100 \mathrm{mg} / \mathrm{ml}$ streptomycin were used to culture cells. Cells were incubated at $37^{\circ} \mathrm{C}$ with the $5 \%$ concentration of $\mathrm{CO}_{2}$.

\section{Transcriptome data download and pre-processing}

We downloaded the transcriptome RNA-sequencing data of LUAC samples from TCGA data portal (https://portal.gdc.cancer.gov/). Besides, we obtained the clinical parameters of these patients and excluded patients with the poor overall survival (less than 30 days) to decrease the non-cancer-specific mortality. These data were current updated in April 07, 2020. The Perl language (http://www.perl.org/) was used to process RNA-seq data and clinical outcomes.

\section{IRLNRs extraction}

IRGs were identified by The Molecular Signatures Database v4.0 (http://www.broadinstitute.org/gsea/m sigdb/index.jsp) (Supplementary Files 1, 2). IRGs were utilized to calculate the immune-related score of LUAC through GSEA. The correlations between immunerelated score and LncRNAs expression were analyzed through Pearson correlation analysis. IRLNRs need to conform to the standard of $|\mathrm{r}|>0.4$ and $P<0.001$.

\section{Acquire the survival-related IRLNRs (sIRLNRs)}

IRLNRs with correlation of OS were regarded as sIRLNRs in LUAC patients. The sIRLNRs were identified by univariate $\mathrm{COX}$ regression analysis $(\mathrm{P}<0.001)$. Besides, the sIRLNRs were divided into protective and deleterious portion by Hazard ratio.

\section{Immune-related risk score model creation}

Following multivariate COX regression analysis, the integrated sIRLNRs were regarded as independent prognostic factors to establish the immune-related risk score model (IRRS). IRRS was created based on the expression level of sIRLNRs multiplied by 
the coefficients of Cox regression. [AC068338.3 $*(-0.45635)]+[$ AL691432.2 * $(-0.25455)]+$ [AL606489.1* (0.210469)] + [TMPO-AS1* (0.473489)] $+[$ AP000695.1* $(0.358633)]$. We classify patients with LUAC into the high-risk group and the low-risk group using the median score. Besides, we obtained the immune cell infiltration status of LUAC patients, and analyzed their relationships with the IRRS.

\section{Bioinformatics analysis}

Based on the IRRS, the sensitivity and specificity were evaluated via ROC curve. Principal component analysis (PCA) and GSEA was displayed to explore the differential expression levels and phenotypes between patients with various risks. Kaplan-Meier curve was utilized to assess the OS. The independent prognostic factors of LUAC patients were identified by Cox regression analysis. The nomogram was employed to predict the survival probabilities.

\section{Real-time quantitative PCR}

The real-time quantitative PCR (qPCR) was conducted as previous report [30]. Extracted total RNA from tissues and cell lines by Triazole (Invitrogen). The reverse transcribed cDNA was obtained by cDNA Synthesis Kit (Osaka, Japan of TaKaRa). The qPCR was conducted on an ABI 7500 real-time PCR system (Applied Biosystems). The primer sequences are illustrated in Table 1. Each cDNA sample was analyzed three times.

\section{Statistical analysis}

Statistical analysis was performed by SPSS 21.0 software (Chicago, IL, USA) and GraphPad Prism5 (La Jolla, CA, USA). Varieties in clinical parameters were validated using ANOVA, post-hoc test and independent T-test. $P<0.05$ was considered significantly statistical difference.

\section{Availability of data and materials}

Authors can provide all of datasets analyzed during the study on reasonable request.

\section{Ethics approval and consent to participate}

Informed consent forms have been signed by all patients before this study. The research protocol has been approved by the Ethics Committee of Chongqing University Cancer Hospital and is based on the ethical principles of medical research involving human subjects in the Helsinki Declaration.

\section{RESULTS}

\section{IRLNRs acquisition}

Transcriptome RNA-sequencing data and clinical parameters were downloaded from TCGA database. Following that, LncRNAs and mRNAs data were extracted from transcriptome data. We screened 331 IRGs, of which 36 LncRNAs were identified to be the IRLNRs by correlation analysis.

\section{Correlation between prognosis of LUAC patients and IRLNRs}

Following the univariate COX regression analysis, we then validated 10 IRLNRs which were associated with prognoses of LUAC patients, including FAM83A-AS1, AC022613.1, AC068338.3, AL691432.2, HSPC324, AC087752.3, AL606489.1, TMPO-AS1, AP000695.1 and AL034397.3 $(\mathrm{P}<0.001)$. The correlation between the sIRLNRs and prognosis was shown in the forest plot (Figure 1).

\section{Prognostic features of different risk groups}

The five sIRLNRs (AC068338.3, AL691432.2, AL606489.1, TMPO-AS1 and AP000695.1) among the 10 sIRLNRs were selected by multivariate COX regression analysis, and were utilize to develop the IRRS, by which the LUAC patients were separated into the high-risk group and the low-risk group (Figure 2A). We found that the mortality rate constantly increased with the higher risk score (Figure 2B). With the increase of the risk score, the expression levels of AL606489.1, TMPO-AS1 and AP000695.1 were increased, while AC068338.3 and AL691432.2 were decreased (Figure 2C). Furthermore, as illustrated in Figure 3, compared to the low-risk group, the survival curve of patients in the high-risk group was remarkably lower.

\section{Correlation between IRRS and clinical features}

To explore the clinical significances of the sIRLNRs, we analyzed the relevance of IRRS and clinicopathologic features of LUAC. We found that the expression levels of AC068338.3 and AL691432.2 were lower in LUAC patients with advanced stages and Tstages (Figure 4A, 4B). Besides, the expression of AP000695.1 was higher with the more advanced Nstages, while AC068338.3 and AL691432.2 showed reversed variations (Figure 4C). Additionally, we didn't observe remarkable differences in various M-stages, and it was probably due to the limited samples size of distant metastasis (Figure 4D). We further displayed univariate $\mathrm{COX}$ regression analysis, and we found that stage, T-stage, $\mathrm{N}$-stage, and risk score were prominently 
Table 1. The primer sequences of AC068338.3 and AL691432.2.

\begin{tabular}{cll}
\hline \multirow{2}{*}{$\mathbf{A C 0 6 8 3 3 8 . 3}$} & F primer (5'-3') & AGGCTCGGGAGTGTGATTTG \\
& R primer (5'-3') & CTTTGGTGCAGTGTTTCCGG \\
AL691432.2 & F primer (5'-3') & TTTGGACCAAAGGCCTGTGT \\
& R primer (5'-3') & CAACTCCACACACATCCCGA \\
\multirow{3}{\beta}{-actin } & F primer (5'-3') & AAACGTGCTGCTGACCGAG \\
& R primer (5'-3') & TAGCACAGCCTGGATAGCAAC \\
\hline
\end{tabular}

Note: F primer, forward primer; R primer, reverse primer.

associated with OS. Nevertheless, in the multivariate COX regression analysis, only stage and risk score illustrated remarkable correlation with OS (Table 2). Then, the ROC curve was employed to evaluate the accuracy of IRRS. As shown in the Figure 5, the AUC of risk score, age, gender, stage, T-stage, M-stage and $\mathrm{N}$-stage were $0.756,0.511,0.586,0.729,0.666,0.495$ and 0.680 separately. To further validate the clinical significance and values of IRRS, we drew a nomogram of LUAC by multivariate COX analysis results of the 5 sIRLNRs in the IRRSM (Figure 6). The nomogram will provide another reference for clinical decision-makers to evaluate the prognosis of LUAC patients. These results suggested that risk score is an independent prognostic factor of LUAC.

\begin{tabular}{|c|c|}
\hline pvalue & Hazard ratio \\
\hline FAM83A-ASK0.001 & $1.274(1.104-1.471)$ \\
\hline AC022613.1<0.001 & $1.432(1.161-1.768)$ \\
\hline AC068338.3<0.001 & $0.383(0.233-0.628)$ \\
\hline AL691432. $2<0.001$ & $0.582(0.441-0.768)$ \\
\hline HSPC324 <0.001 & $0.433(0.269-0.696)$ \\
\hline AC087752.3<0.001 & $0.502(0.333-0.757)$ \\
\hline AL606489.1<0.001 & $1.664(1.290-2.148)$ \\
\hline TMPO-AS1 <0.001 & $1.820(1.332-2.487)$ \\
\hline AP000695.1<0.001 & $1.807(1.340-2.436)$ \\
\hline AL034397.3<0.001 & $0.574(0.416-0.791)$ \\
\hline
\end{tabular}

\section{The immune status of different risk groups}

In the immune-related risk gene sets, we found that, compared to the low-risk group, the high-risk group obtained the higher immune score (Figure 7A). However, based on the genome-wide expression profiles, the prominent separation between the two risk groups wasn't observed (Figure 7B).

The expression of AC068338.3 and AL691432.2 were lower in LUAC cell lines and LUAC patients especially with advanced $\mathrm{T}$-stages

To validate the expression levels of sIRLNRs in vivo and in vitro, we examined the expression of

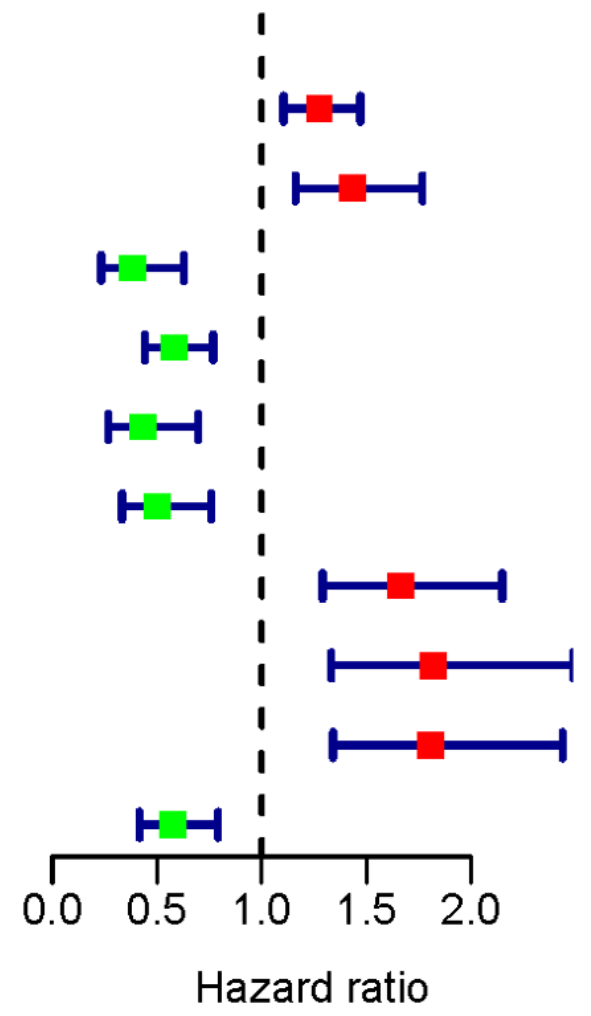

Figure 1. Survival-related IRLNRs forest plot. The hazard ratios of survival-related IRLNRs (FAM83A-AS1, AC022613.1, AC068338.3, AL691432.2, HSPC324, AC087752.3, AL606489.1, TMPO-AS1, AP000695.1 and AL034397.3) were illustrated in Forest plot (P<0.001). Red parts represent upregulated sIRLNRs, and green parts represent downregulated sIRLNRs. 
AC068338.3 and AL691432.2 in bronchial epithelial cells, different LUAC cell lines, tumor tissues, adjacent normal tissues and tumor samples with different T-stages. We found that, compared to HBE, the expression of AC068338.3 and AL691432.2 were significantly lower in A549, HCC827, NCIH1299, NCI-H2228 and NCI-H1975 cell lines (Figure 8A). Besides, we detected the remarkably lower expression levels of AC068338.3 and AL691432.2 in tumor tissues than those in adjacent tissues, (Figure 8B) and the expression levels of AC068338.3 and AL691432.2 in T1 and T2 stages were remarkably higher than those in more advanced T-stages (Figure 8C).

\section{DISCUSSION}

Although radical resection is an effective treatment option for LUAC patients, the high recurrence rate after operation and some advanced-stages patients with unresectable tumors are still the great challenges [8]. Therefore, researchers are paying attentions to explore other appropriate treatment strategies for the group of patients. With the in-depth understanding of immune

\section{A}

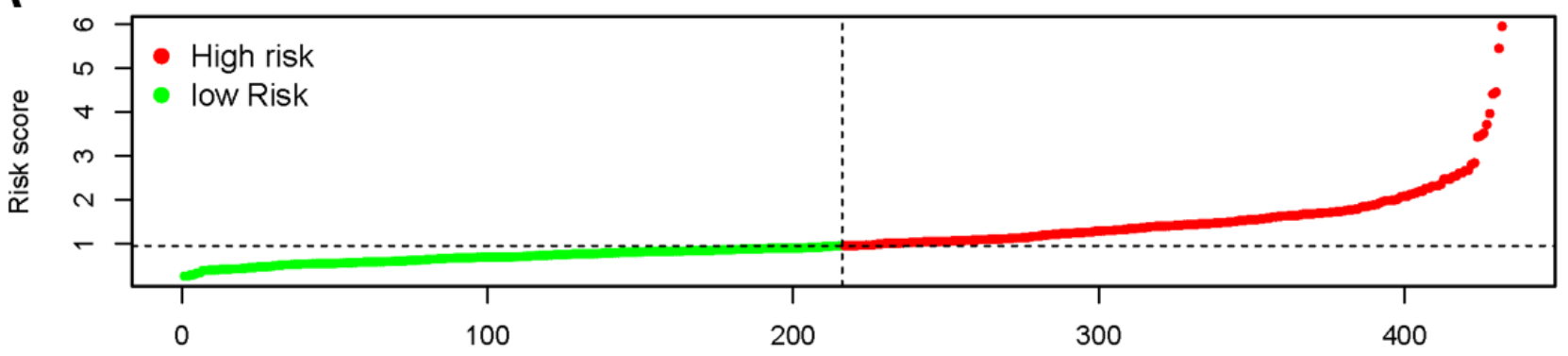

B

Patients (increasing risk socre)

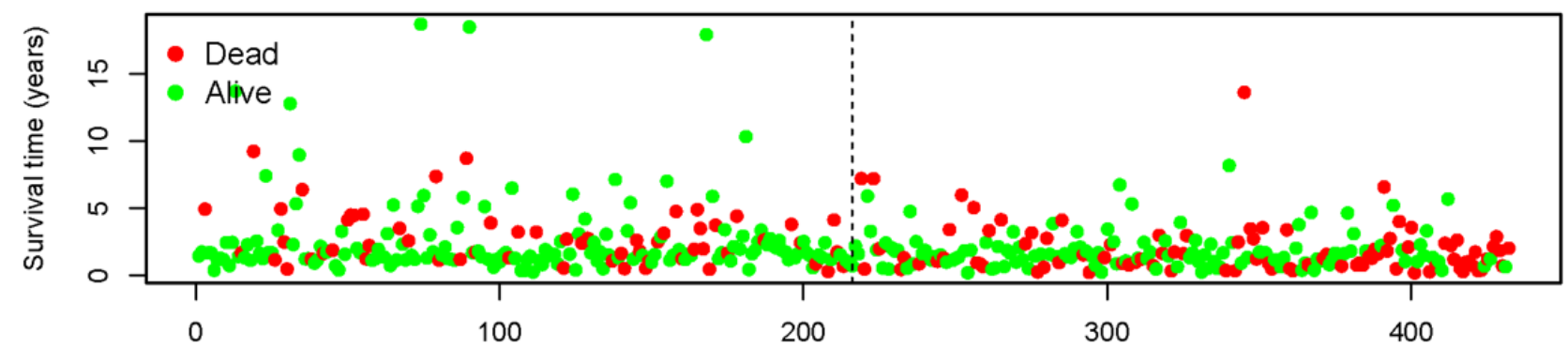

C

Patients (increasing risk socre)

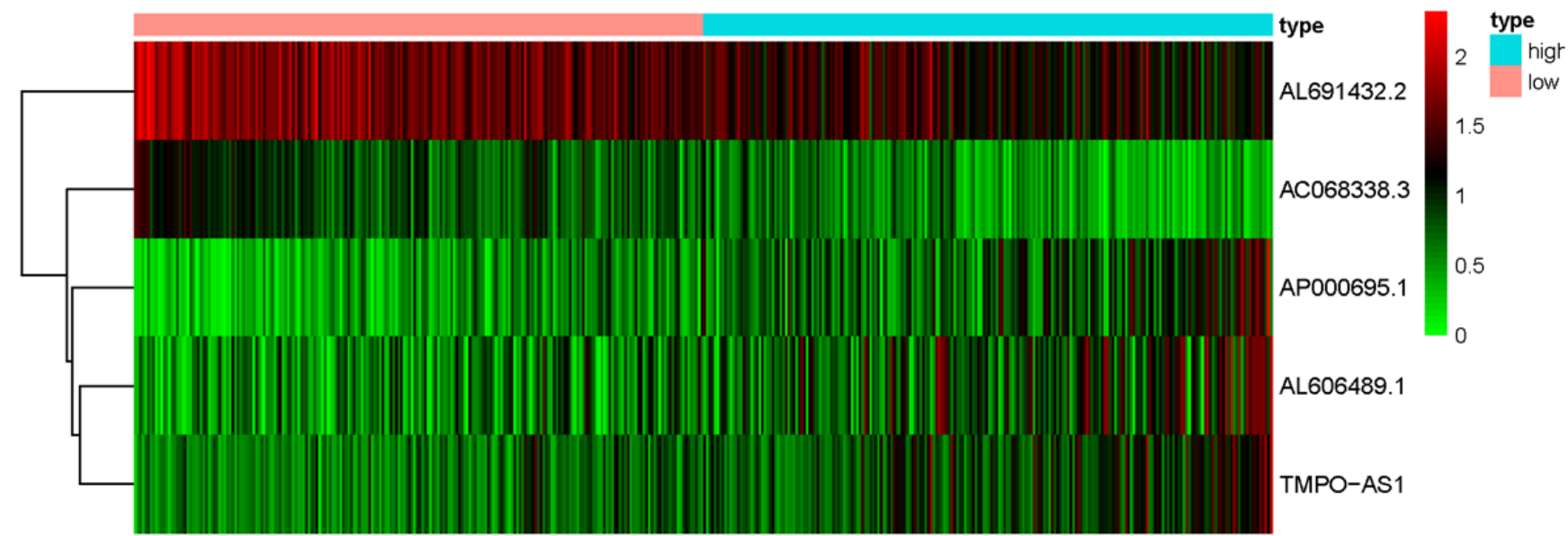

Figure 2. Immune-related risk score model (IRRS) was established according to sIRLNRs. The risk score distributions of high-risk group and low-risk group (A). Survival status between high-risk group and low-risk group (B). The expression levels of sIRLNRs in the heatmap (C). 


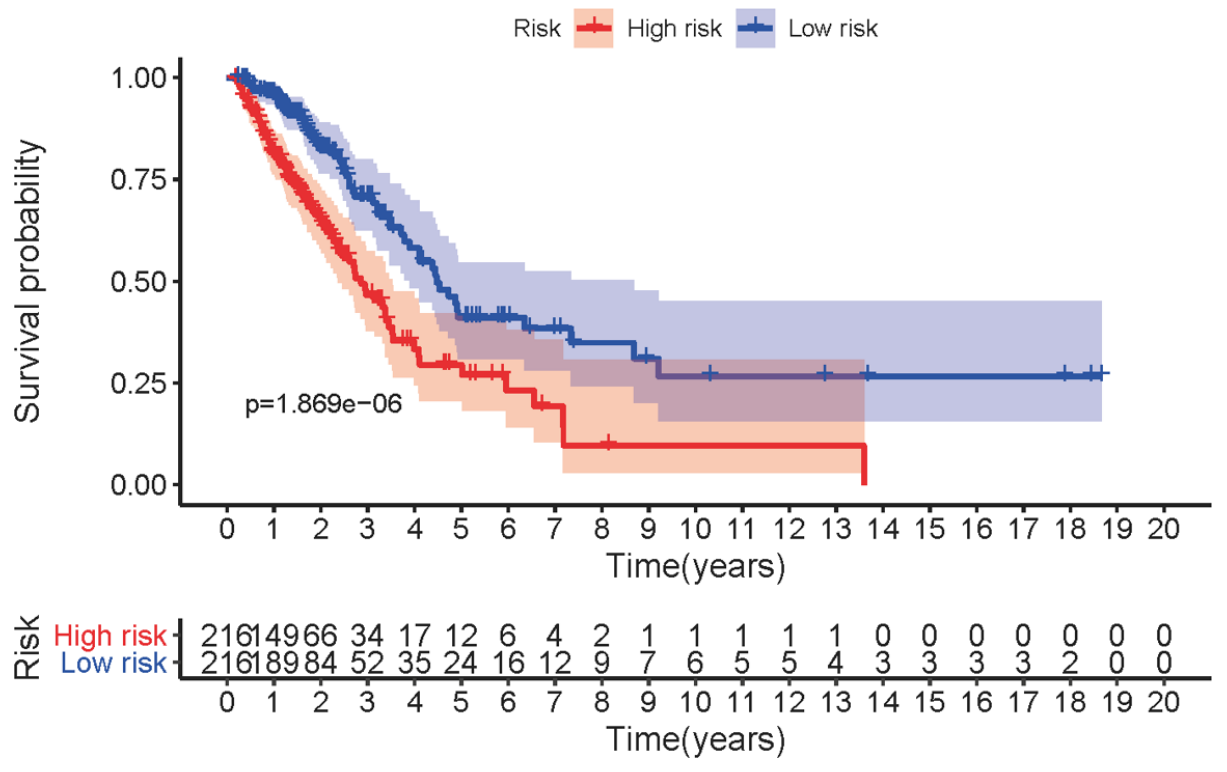

Figure 3. Survival curve of LUAC patients. Kaplan-Meier survival curve of OS in high-risk group and low-risk group. The results showed that the high-risk group have the poor prognosis.

A

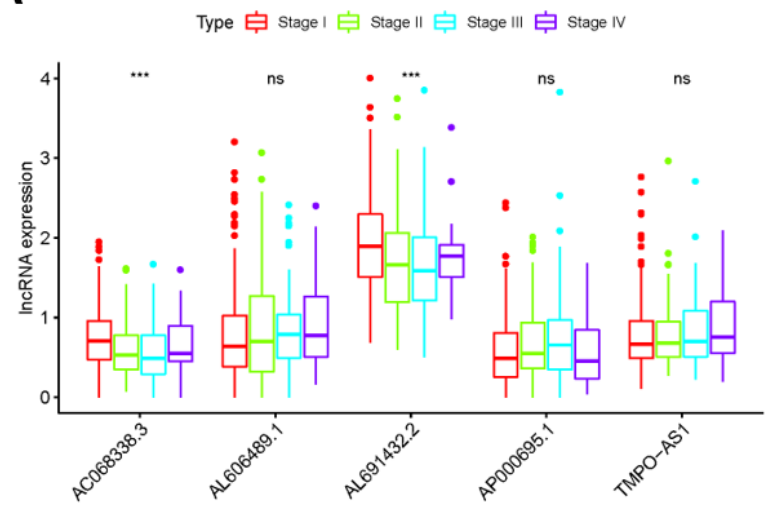

C

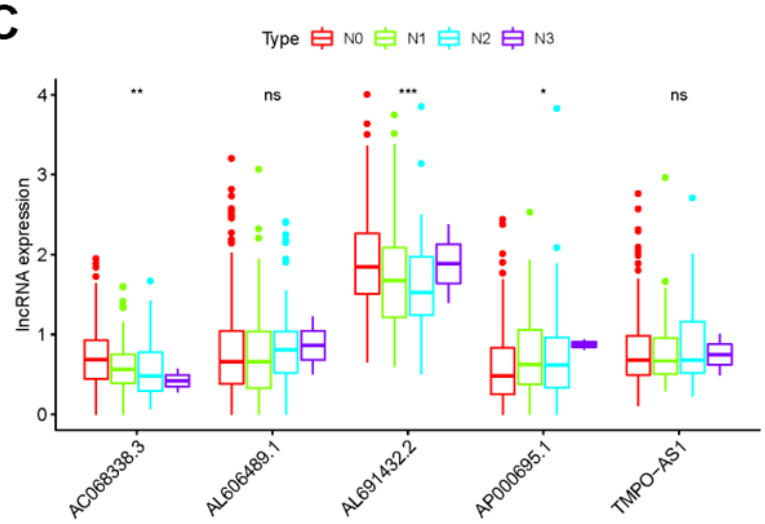

B

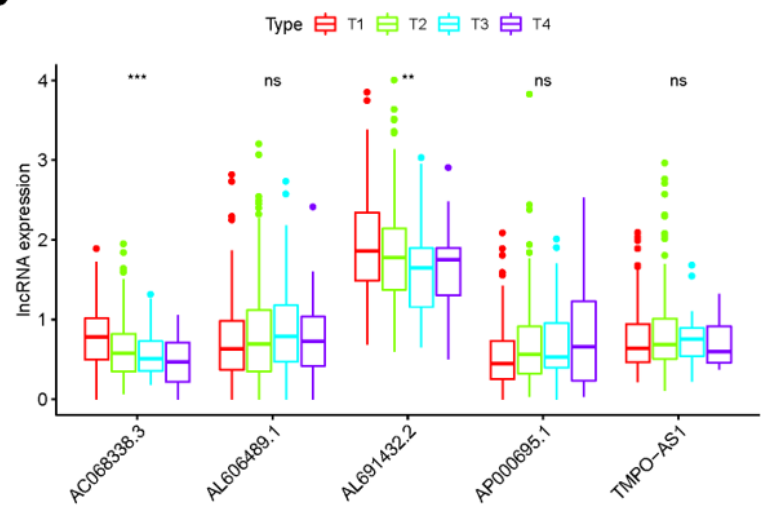

D $\quad$ Type 白 mo 白 m1 $^{2}$

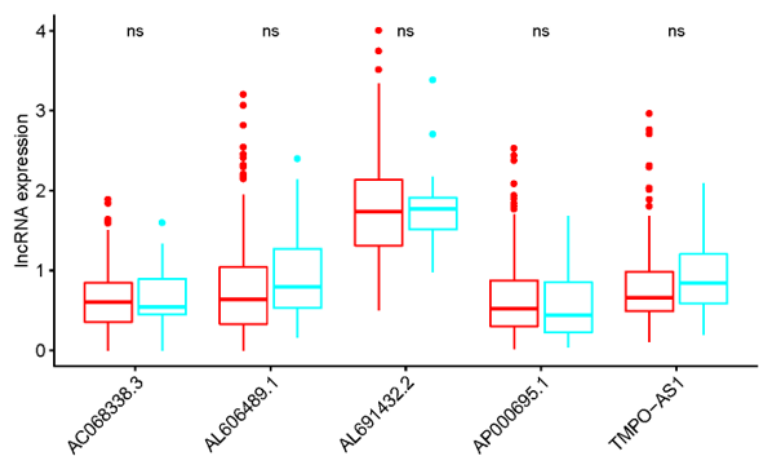

Figure 4. The relationships between the sIRLNRs and clinical features. Relationships between sIRLNRs (AC068338.3, AL691432.2, AL606489.1, TMPO-AS1 and AP000695.1) and clinical features. The expression levels of AL606489.1, TMPO-AS1 and AP000695.1 were increased in the more advanced stages (A) and T-stages (B), while the expression levels of AC068338.3 and AL691432.2 were gradually decreased. The expression levels of AC068338.3 and AL691432.2 were decreased in the more advanced N-stages (C), while the expression levels of AP000695.1 were increased. There was no significant difference in various $M$-stages. (D) $\left({ }^{* * *} P<0.001 ;{ }^{* *} P<0.01 ;{ }^{*} P<0.05 ; n\right.$ : $\left.P>0.05\right)$. 
Table 2. Univariate and multivariate analysis of LUAC.

\begin{tabular}{lcccccccc}
\hline \multirow{2}{*}{ Variables } & \multicolumn{3}{c}{ Univariate analysis } & \multicolumn{3}{c}{ Multivariate analysis } \\
\cline { 2 - 8 } & HR & $\begin{array}{c}\text { HR 95\% } \\
\text { low }\end{array}$ & $\begin{array}{c}\text { HR 95\% } \\
\text { high }\end{array}$ & P value & HR & $\begin{array}{c}\text { HR 95\% } \\
\text { low }\end{array}$ & $\begin{array}{c}\text { HR 95\% } \\
\text { high }\end{array}$ & P value \\
\hline Age & 1.001956 & 0.982501 & 1.021797 & 0.845098 & 1.011486 & 0.991531 & 1.031843 \\
Gender & 1.033875 & 0.711617 & 1.502069 & 0.861233 & 0.923619 & 0.631409 & 1.351062 & 0.261273 \\
Stage & 1.645457 & 1.390237 & 1.947529 & $6.98 \mathrm{E}-09$ & 2.03528 & 1.235723 & 3.352179 & 0.005249 \\
T-stage & 1.589128 & 1.272484 & 1.984567 & $4.40 \mathrm{E}-05$ & 1.11824 & 0.875036 & 1.429038 \\
M-stage & 1.661335 & 0.888927 & 3.104904 & 0.111621 & 0.32111 & 0.084868 & 1.214957 & 0.371786 \\
N-stage & 1.784283 & 1.448821 & 2.197419 & $5.06 \mathrm{E}-08$ & 0.939858 & 0.611679 & 1.444112 & 0.777149 \\
Risk score & 1.684247 & 1.423866 & 1.992243 & $1.17 \mathrm{E}-09$ & 1.570501 & 1.308105 & 1.885531 & $1.30 \mathrm{E}-06$ \\
\hline
\end{tabular}

Note: HR, Hazard Ratio.

activities in the occurrence, development and prognosis of tumor, a growing body of promising immunotherapies provide new hopes for LUAC patients [31-33]. Up to now, immunotherapy has effectively prolonged the survival time of a large number of patients, but another part of patients showed limited responses [34]. Multiplied studies indicated the individual difference of the gene levels might take responsibility $[35,36]$.

In the past few years, series of studies have illustrated immune cell infiltration plays the crucial role in the prognosis of patients with LUAC [18, 37-39]. The researchers also highlighted the effect of tumor immune activity on clinical decision-making [40, 41]. Therefore, the roles of gene expression levels on tumor immune activities were deserved to be further explored. Kaiyong Yang reported that Angiotensin II contributed to immunosuppression by induction of PD-L1 expression in NSCLC [42]. Heidi Dvinge indicated that the significant function of miRNA in predicting the prognosis of breast cancer patients [43]. Serum microRNA expression could predict the survival of NSCLC [44]. Overexpression of LncRNA MALAT-1 has been demonstrated to have potential for predicting the recurrence of hepatocellular carcinoma after liver transplantation [24].

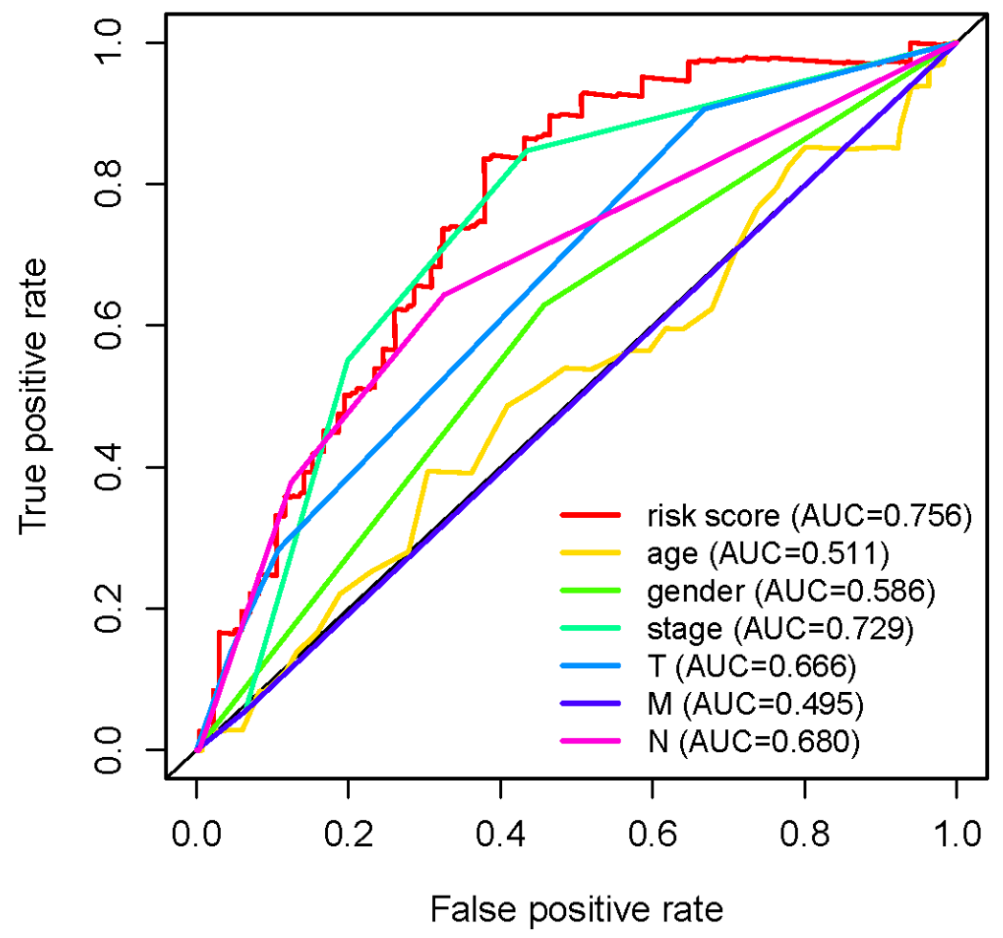

Figure 5. Receiver operating characteristic (ROC) curve. The prognostic value of the independent prognostic factors was indicated by ROC curves. 
Currently, judging the prognosis of patients with tumor by IRGs has also become a research focus [45, 46]. Song Qian has demonstrated IRGs could be served as biomarkers to predict the prognosis of LUAC [47]. In the field of immune microenvironment, emerging evidence has suggested that IncRNAs are closely involved in the regulation of immune autoimmunity and function [48]. LncRNAs have been ascertained to participate in regulating various important immune processes, such as the production of inflammatory mediators, cells differentiation and migration [49]. Xu et al. reported that SATB2-AS1 can

Points

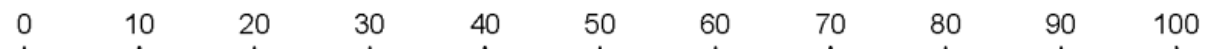

AC068338.3

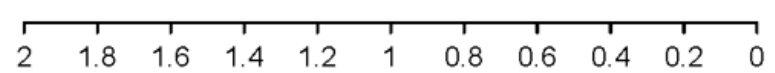

AL691432.2

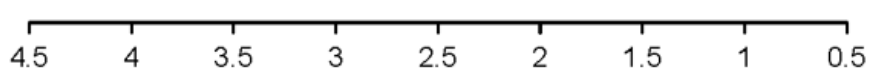

AL606489.1

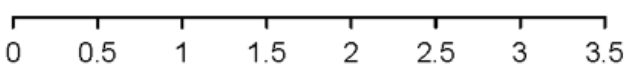

TMPO-AS1

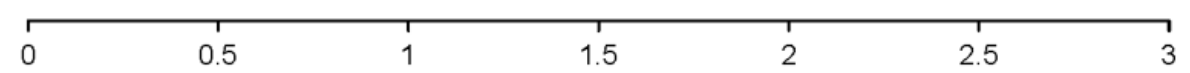

AP000695.1

Total Points

\begin{tabular}{llllllll}
\hline 0 & 0.5 & 1 & 1 & 1 & 3 & 3.5 & 4
\end{tabular}

1-year survival

\begin{tabular}{|c|c|c|c|c|c|c|}
\hline$\Gamma$ & $T$ & $T$ & $T$ & 1 & 1 & $\longrightarrow$ \\
\hline 0 & 50 & 100 & 150 & 200 & 250 & 300 \\
\hline
\end{tabular}

3-year survival

5-year survival

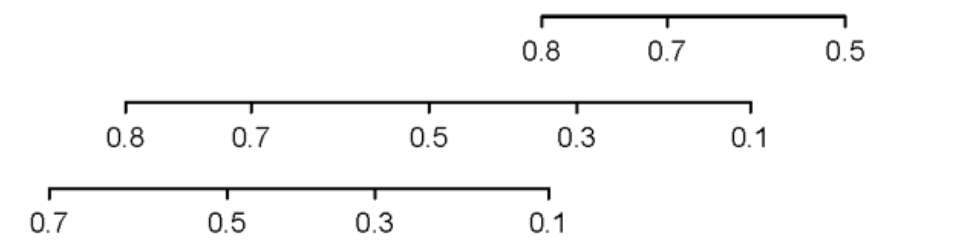

Figure 6. Nomogram of LUAC patients. Nomogram was used to predict 1, 3, and 5-year survival rates for LUAC patients.

A

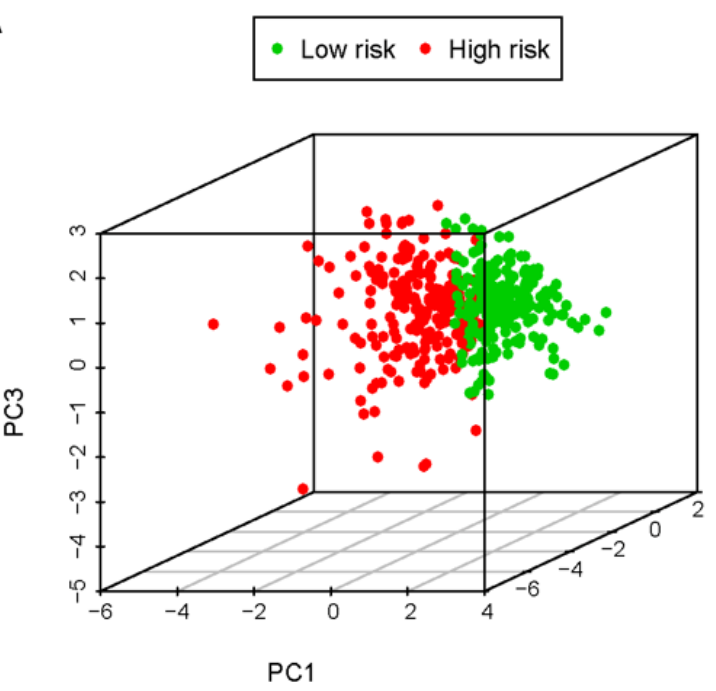

B

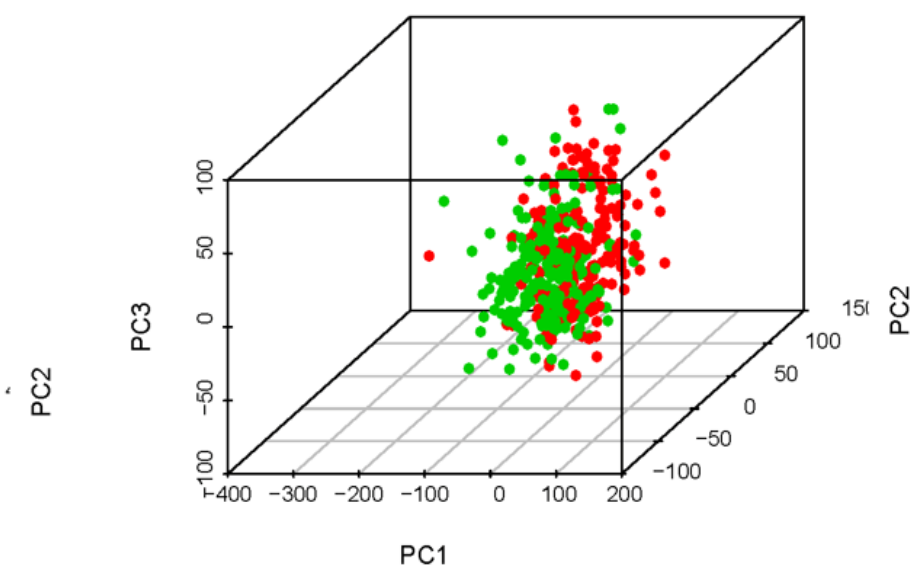

Figure 7. The principal components analysis (PCA). The high-risk group and low-risk group tended to express different immune status. PCA among high-risk group and low-risk group based on the immune-related risk gene sets (A). PCA among high-risk group and low-risk group based on the genome-wide expression sets (B). The results showed that the high-risk group and low-risk group were divided into two parts in immune-related risk gene sets. 
regulate the expression of TH1 chemokine and the density of immune cells in CRC to inhibit the progression of CRC [50]. But the mechanism of IRLNRs on the prognosis of patients with LUAC has not been fully elucidated.

In this study, we analyzed TCGA LncRNAs data of 533 patients with LUAC, and screened 311 IRGs, of which 36 IRLNRs were identified as IRLNRs. Besides, we found 10 IRLNRs with prominent relevance to OS. Five IRLNRs were further identified to create an IRRS, by which LUAC patients with different risk scores were distinguished. Following the multivariate COX analysis, we found that risk score is an independent prognostic factor to assess the prognosis of LUAC patients. Given that the low purity of tumor and the infiltration of immune cell components were closely associated with the tumor development, immunophenotype and prognosis $[51,52]$, we therefore used PCA to explore the differential distribution of different risk groups, and we observed the lower immune score in patients with the lower risk. Besides, neutrophil cells were enriched in the high-risk group, but B cells were enriched in the low-risk group. Therefore, we think that IRRS is significantly related to the immune status of LUAC patients.

Additionally, to increase the reliability of clinical evidences, a great number of patients with LUAC were recruited in the present study. To further validate the correlation between sIRLNRs and tumor clinical features, we examined the expression of AC068338.3 and AL691432.2 in vitro and in vivo. These results suggested that IRRS based on the five sIRLNRs can help doctors to identify LUAC patients with the similar clinicopathologic and molecular characteristics, and then contribute to the more precise treatment strategies.

We have verified the expression levels of some sIRLNRs (AC068338.3 and AL691432.2) of IRRS in LUAC cell lines and tumor tissues and adjacent tissues of LUAC patients. As for other 3 lncRNAs which were
A

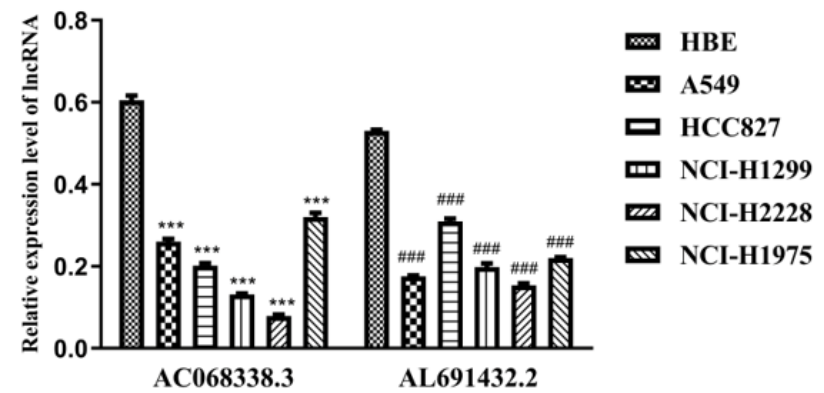

C

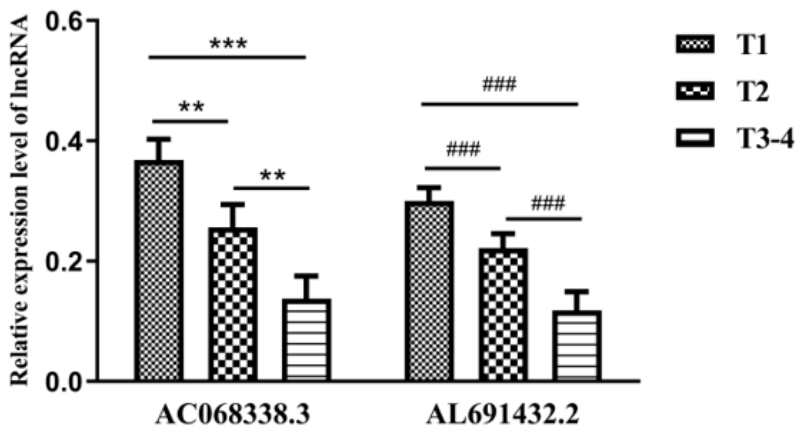

B

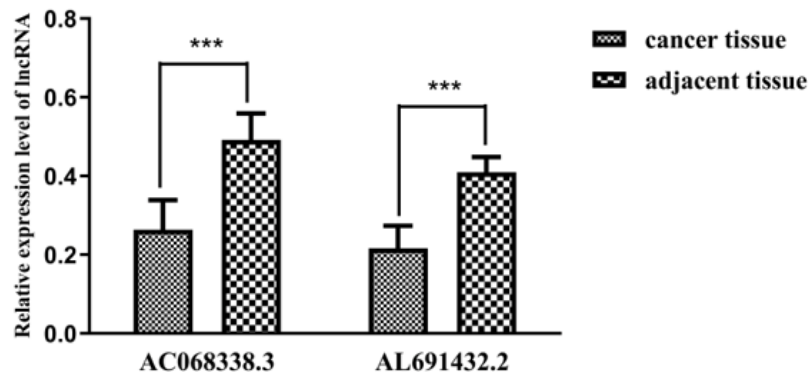

Figure 8. The expression levels of two sIRLNRs and the relationships with various T-stages. The results of RT-qPCR of the expression levels of AC068338.3 and AL691432.2 in LUAC cell lines and tumor and adjacent tissues. The expression levels of AC068338.3 and AL691432.2 in HBE were both higher than those in LUAC cell lines, *** represents the significant difference of AC068338.3 compared with $\operatorname{HBE}(\mathrm{P}<0.001)$, \#\#\# represents the significant difference of AL691432.2 compared with HBE $(P<0.001)(A)$. Compared with cancer tissues, the expression levels of AC068338.3 and AL691432.2 were both higher in adjacent tissues; ${ }^{* * *}$ represents the significant difference of AC068338.3 ( $\mathrm{P}<0.001)$, \#\# represents the significant difference of AL691432.2 ( $<<0.001)(B)$. The expression levels of AC068338.3 and AL691432.2 were both the lowest in LUAC patients of T3-4 stages, and the highest expression levels of AC068338.3 and AL691432.2 were both detected in T1 patients; ${ }^{* * *}$ represents the significant difference of AC068338.3 $(\mathrm{P}<0.001),{ }^{* *}$ represents the significant difference of AC068338.3 ( $P<0.01)$, \#\# represents the significant difference of AL691432.2 $(+<0.001)$ (C). 
not further validated in the study, we found that TMPOAS1 plays remarkable roles in promoting tumorigenesis and development of bladder cancer, liver cancer, cervical cancer and lung cancer $[15,26,53]$. In Mu's study, TMPO-AS1 was demonstrated to promote lung adenocarcinoma progression by targeting miR-383-5p [54]. Besides, AL606489.1 was demonstrated to be regulate ferroptosis and autophagy [28, 29]. Few reports have studied the role of AP000695.1 in cancer, so more focus need to be turned toward the effect and underlying mechanism of AP000695.1 in LUAD.

\section{CONCLUSIONS}

In this study, we identify and validate the LUACrelated sIRLNRs with prominent clinical significances, and create a reliable risk assessing model based on the sIRLNRs to assess prognosis of LUAC patients. The results will offer a novel perspective for immunerelated studies and immunotherapeutic strategies of LUAC.

\section{Abbreviations}

LCa: lung cancer; LUAC: lung adenocarcinoma; TCGA: The Cancer Genome Atlas; GSEA: gene set enrichment analysis; PCA: principal component analysis; TME: tumor microenvironment; IME: immune microenvironment; IRGs: immune-related genes; IRLNRs: immune related long non-coding RNAs; IRRS: immune-related risk score model; LncRNAs: long non-coding RNAs; sIRLNRs: survival-related IRLNRs; OS: overall survival.

\section{AUTHOR CONTRIBUTIONS}

This work was carried out with collaboration among all authors. Yiming Wang and Hao Long contributed to allround consideration of the study. Songmei Lu performed the experiments and wrote parts of the manuscript. Nan Shan wrote the manuscript. Xingyue Chen and Fangliang Peng collected the samples and analyzed data.

\section{CONFLICTS OF INTEREST}

The authors declare that they have no conflicts of interest.

\section{FUNDING}

The study is funded by the program of Chongqing Science and Technology Bureau (Research on the mechanism of SRC-3 regulating JAK/STAT signaling pathway through acetylation modification in the development and progression of colorectal cancer, cstc2019jcyj-msxmX0721).

\section{REFERENCES}

1. de Koning HJ, van der Aalst CM, de Jong PA, Scholten ET, Nackaerts K, Heuvelmans MA, Lammers JJ, Weenink C, Yousaf-Khan U, Horeweg N, van 't Westeinde S, Prokop M, Mali WP, et al. Reduced LungCancer Mortality with Volume CT Screening in a Randomized Trial. N Engl J Med. 2020; 382:503-13.

https://doi.org/10.1056/NEJMoa1911793 PMID:31995683

2. Yang D, Liu Y, Bai C, Wang X, Powell CA. Epidemiology of lung cancer and lung cancer screening programs in China and the United States. Cancer Lett. 2020; 468:82-7.

https://doi.org/10.1016/i.canlet.2019.10.009 PMID: $\underline{31600530}$

3. Thai AA, Solomon BJ, Sequist LV, Gainor JF, Heist RS. Lung cancer. Lancet. 2021; 398:535-54. https://doi.org/10.1016/S0140-6736(21)00312-3 PMID:34273294

4. Krist AH, Davidson KW, Mangione CM, Barry MJ, Cabana M, Caughey AB, Davis EM, Donahue KE, Doubeni CA, Kubik M, Landefeld CS, Li L, Ogedegbe G, et al, and US Preventive Services Task Force. Screening for Lung Cancer: US Preventive Services Task Force Recommendation Statement. JAMA. 2021; 325:962-70.

https://doi.org/10.1001/jama.2021.1117

PMID: $\underline{3687470}$

5. Zhang $\mathrm{H}$, Jiang $\mathrm{H}$, Zhu L, Li J, Ma S. Cancer-associated fibroblasts in non-small cell lung cancer: Recent advances and future perspectives. Cancer Lett. 2021; 514:38-47.

https://doi.org/10.1016/j.canlet.2021.05.009 PMID: $\underline{34019960}$

6. Warth A, Muley T, Meister M, Stenzinger A, Thomas M, Schirmacher P, Schnabel PA, Budczies J, Hoffmann H, Weichert $W$. The novel histologic International Association for the Study of Lung Cancer/American Thoracic Society/European Respiratory Society classification system of lung adenocarcinoma is a stage-independent predictor of survival. J Clin Oncol. 2012; 30:1438-46.

https://doi.org/10.1200/JCO.2011.37.2185

PMID:22393100

7. Duffy MJ, O'Byrne K. Tissue and Blood Biomarkers in Lung Cancer: A Review. Adv Clin Chem. 2018; 86:1-21. https://doi.org/10.1016/bs.acc.2018.05.001 PMID:30144837

8. Chemi F, Rothwell DG, McGranahan N, Gulati S, Abbosh C, Pearce SP, Zhou C, Wilson GA, Jamal-Hanjani M, Birkbak N, Pierce J, Kim CS, Ferdous S, et al, and TRACERx Consortium. Pulmonary venous circulating 
tumor cell dissemination before tumor resection and disease relapse. Nat Med. 2019; 25:1534-9.

https://doi.org/10.1038/s41591-019-0593-1

PMID:31591595

9. Patel SP, Kurzrock R. PD-L1 Expression as a Predictive Biomarker in Cancer Immunotherapy. Mol Cancer Ther. 2015; 14:847-56.

https://doi.org/10.1158/1535-7163.MCT-14-0983 PMID:25695955

10. Ellinger J, Alam J, Rothenburg J, Deng $M$, Schmidt D, Syring I, Miersch $\mathrm{H}$, Perner S, Müller SC. The long noncoding RNA Inc-ZNF180-2 is a prognostic biomarker in patients with clear cell renal cell carcinoma. Am J Cancer Res. 2015; 5:2799-807.

PMID:26609485

11. Rittmeyer A, Barlesi F, Waterkamp D, Park K, Ciardiello F, von Pawel J, Gadgeel SM, Hida T, Kowalski DM, Dols MC, Cortinovis DL, Leach J, Polikoff J, et al, and OAK Study Group. Atezolizumab versus docetaxel in patients with previously treated non-small-cell lung cancer (OAK): a phase 3, open-label, multicentre randomised controlled trial. Lancet. 2017; 389:255-65. https://doi.org/10.1016/S0140-6736(16)32517-X PMID:27979383

12. Cabel L, Riva F, Servois V, Livartowski A, Daniel C, Rampanou A, Lantz O, Romano E, Milder M, Buecher B, Piperno-Neumann S, Bernard V, Baulande S, et al. Circulating tumor DNA changes for early monitoring of anti-PD1 immunotherapy: a proof-of-concept study. Ann Oncol. 2017; 28:1996-2001.

https://doi.org/10.1093/annonc/mdx212 PMID:28459943

13. Jain RK. Normalizing tumor microenvironment to treat cancer: bench to bedside to biomarkers. J Clin Oncol. 2013; 31:2205-18.

https://doi.org/10.1200/JCO.2012.46.3653

PMID:23669226

14. Zhao H, Yang L, Baddour J, Achreja A, Bernard V, Moss T, Marini JC, Tudawe T, Seviour EG, San Lucas FA, Alvarez H, Gupta S, Maiti SN, et al. Tumor microenvironment derived exosomes pleiotropically modulate cancer cell metabolism. Elife. 2016; 5:e10250. https://doi.org/10.7554/eLife.10250 PMID:26920219

15. Wang Z, Huang D, Huang J, Nie K, Li X, Yang X. IncRNA TMPO-AS1 Exerts Oncogenic Roles in HCC Through Regulating miR-320a/SERBP1 Axis. Onco Targets Ther. 2020; 13:6539-51.

https://doi.org/10.2147/OTT.S250355 PMID:

16. Mlecnik B, Bindea G, Angell HK, Maby $P$, Angelova $M$, Tougeron D, Church SE, Lafontaine L, Fischer M, Fredriksen T, Sasso M, Bilocq AM, Kirilovsky A, et al.
Integrative Analyses of Colorectal Cancer Show Immunoscore Is a Stronger Predictor of Patient Survival Than Microsatellite Instability. Immunity. 2016; 44:698-711.

https://doi.org/10.1016/j.immuni.2016.02.025

PMID:26982367

17. Zhou C, Cheng X, Tu S. Current status and future perspective of immune checkpoint inhibitors in colorectal cancer. Cancer Lett. 2021; 521:119-29.

https://doi.org/10.1016/i.canlet.2021.07.023 PMID:34464671

18. Sun J, Zhang Z, Bao S, Yan C, Hou P, Wu N, Su J, Xu L, Zhou M. Identification of tumor immune infiltrationassociated IncRNAs for improving prognosis and immunotherapy response of patients with non-small cell lung cancer. J Immunother Cancer. 2020; 8:e000110.

https://doi.org/10.1136/iitc-2019-000110

PMID:32041817

19. Gu Z, Du Y, Zhao X, Wang C. Tumor microenvironment and metabolic remodeling in gemcitabine-based chemoresistance of pancreatic cancer. Cancer Lett. 2021; 521:98-108. https://doi.org/10.1016/i.canlet.2021.08.029 PMID:34461181

20. Li $\mathrm{CH}$, Chen Y. Targeting long non-coding RNAs in cancers: progress and prospects. Int J Biochem Cell Biol. 2013; 45:1895-910.

https://doi.org/10.1016/j.biocel.2013.05.030 PMID:23748105

21. Li Y, Li G, Guo X, Yao H, Wang G, Li C. Non-coding RNA in bladder cancer. Cancer Lett. 2020; 485:38-44. https://doi.org/10.1016/i.canlet.2020.04.023 PMID:32437725

22. Gutschner $T$, Hämmerle $M$, Eissmann $M$, Hsu J, Kim $Y$, Hung G, Revenko A, Arun G, Stentrup M, Gross M, Zörnig M, MacLeod AR, Spector DL, Diederichs S. The noncoding RNA MALAT1 is a critical regulator of the metastasis phenotype of lung cancer cells. Cancer Res. 2013; 73:1180-9.

https://doi.org/10.1158/0008-5472.CAN-12-2850 PMID:23243023

23. Mitobe Y, Takayama KI, Horie-Inoue K, Inoue S. Prostate cancer-associated IncRNAs. Cancer Lett. 2018; 418:159-66.

https://doi.org/10.1016/i.canlet.2018.01.012 PMID:29330107

24. Lai MC, Yang Z, Zhou L, Zhu QQ, Xie HY, Zhang F, Wu LM, Chen LM, Zheng SS. Long non-coding RNA MALAT1 overexpression predicts tumor recurrence of hepatocellular carcinoma after liver transplantation. Med Oncol. 2012; 29:1810-6. 
https://doi.org/10.1007/s12032-011-0004-z PMID:21678027

25. Zhang KJ, Tan XL, Guo L. The long non-coding RNA DANCR regulates the inflammatory phenotype of breast cancer cells and promotes breast cancer progression via EZH2-dependent suppression of SOCS3 transcription. Mol Oncol. 2020; 14:309-28.

https://doi.org/10.1002/1878-0261.12622

PMID:31860165

26. Luo $H$, Yang $L$, Liu $C$, Wang $X$, Dong $Q$, Liu L, Wei $Q$. TMPO-AS1/miR-98-5p/EBF1 feedback loop contributes to the progression of bladder cancer. Int J Biochem Cell Biol. 2020; 122:105702.

https://doi.org/10.1016/i.biocel.2020.105702 PMID:32087328

27. Wei L, Liu Y, Zhang H, Ma Y, Lu Z, Gu Z, Ding C. TMPOAS1, a Novel E2F1-Regulated IncRNA, Contributes to the Proliferation of Lung Adenocarcinoma Cells via Modulating miR-326/SOX12 Axis. Cancer Manag Res. 2020; 12:12403-14.

https://doi.org/10.2147/CMAR.S269269

PMID:33293866

28. Guo Y, Qu Z, Li D, Bai F, Xing J, Ding Q, Zhou J, Yao L, Xu Q. Identification of a prognostic ferroptosis-related IncRNA signature in the tumor microenvironment of lung adenocarcinoma. Cell Death Discov. 2021; 7:190. https://doi.org/10.1038/s41420-021-00576-z PMID:34312372

29. Wu L, Wen Z, Song Y, Wang L. A novel autophagyrelated IncRNA survival model for lung adenocarcinoma. J Cell Mol Med. 2021; 25:5681-90. https://doi.org/10.1111/jcmm.16582 PMID:33987935

30. Wang $Y$, Huang L, Shan $N, M a ~ H, ~ L u ~ S$, Chen $X$, Long $H$. Establishing a three-miRNA signature as a prognostic model for colorectal cancer through bioinformatics analysis. Aging (Albany NY). 2021; 13:19894-907.

https://doi.org/10.18632/aging.203400

PMID:34388112

31. Campbell JD, Alexandrov A, Kim J, Wala J, Berger AH, Pedamallu CS, Shukla SA, Guo G, Brooks AN, Murray $\mathrm{BA}$, Imielinski $\mathrm{M}, \mathrm{Hu} \mathrm{X}$, Ling $\mathrm{S}$, et al, and Cancer Genome Atlas Research Network. Distinct patterns of somatic genome alterations in lung adenocarcinomas and squamous cell carcinomas. Nat Genet. 2016; 48:607-16.

https://doi.org/10.1038/ng.3564

PMID:27158780

32. Shi W, Yang X, Xie S, Zhong D, Lin X, Ding Z, Duan S, Mo $F$, Liu A, Yin S, Jiang X, Xu ZP, Lu X. A new PD-1-specific nanobody enhances the antitumor activity of T-cells in synergy with dendritic cell vaccine. Cancer Lett. 2021;
522:184-97.

https://doi.org/10.1016/i.canlet.2021.09.028

PMID:34562519

33. Meng $X$, Liu $Y$, Zhang J, Teng F, Xing L, Yu J. PD-1/PD-L1 checkpoint blockades in non-small cell lung cancer: New development and challenges. Cancer Lett. 2017; 405:29-37.

https://doi.org/10.1016/j.canlet.2017.06.033 PMID:28688973

34. Pfirschke C, Engblom C, Rickelt S, Cortez-Retamozo V, Garris C, Pucci F, Yamazaki T, Poirier-Colame V, Newton A, Redouane Y, Lin YJ, Wojtkiewicz G, Iwamoto $Y$, et al. Immunogenic Chemotherapy Sensitizes Tumors to Checkpoint Blockade Therapy. Immunity. 2016; 44:343-54.

https://doi.org/10.1016/j.immuni.2015.11.024 PMID:26872698

35. Faruki H, Mayhew GM, Serody JS, Hayes DN, Perou CM, Lai-Goldman M. Lung Adenocarcinoma and Squamous Cell Carcinoma Gene Expression Subtypes Demonstrate Significant Differences in Tumor Immune Landscape. J Thorac Oncol. 2017; 12:943-53.

https://doi.org/10.1016/j.jtho.2017.03.010 PMID:28341226

36. $X u$ Y, She $Y$, Li $Y$, Li H, Jia Z, Jiang G, Liang L, Duan L. Multi-omics analysis at epigenomics and transcriptomics levels reveals prognostic subtypes of lung squamous cell carcinoma. Biomed Pharmacother. 2020; 125:109859.

https://doi.org/10.1016/i.biopha.2020.109859 PMID:32036209

37. Lavin $\mathrm{Y}$, Kobayashi S, Leader A, Amir ED, Elefant $\mathrm{N}$, Bigenwald $C$, Remark $R$, Sweeney $R$, Becker $C D$, Levine JH, Meinhof $\mathrm{K}$, Chow A, Kim-Shulze S, et al. Innate Immune Landscape in Early Lung Adenocarcinoma by Paired Single-Cell Analyses. Cell. 2017; 169:750-65.e17.

https://doi.org/10.1016/i.cell.2017.04.014 PMID:28475900

38. McGranahan N, Furness AJ, Rosenthal R, Ramskov S, Lyngaa R, Saini SK, Jamal-Hanjani M, Wilson GA, Birkbak NJ, Hiley CT, Watkins TB, Shafi S, Murugaesu N, et al. Clonal neoantigens elicit $\mathrm{T}$ cell immunoreactivity and sensitivity to immune checkpoint blockade. Science. 2016; 351:1463-9.

https://doi.org/10.1126/science.aaf1490 PMID:26940869

39. Taromi S, Firat E, Simonis A, Braun LM, Apostolova $P$, Elze M, Passlick B, Schumacher A, Lagies S, Frey A, Schmitt-Graeff A, Burger M, Schmittlutz K, et al. Enhanced AC133-specific CAR $T$ cell therapy induces durable remissions in mice with metastatic 
small cell lung cancer. Cancer Lett. 2021; 520: 385-99.

https://doi.org/10.1016/j.canlet.2021.08.012

PMID:34407431

40. Liu X, Wu S, Yang Y, Zhao M, Zhu G, Hou Z. The prognostic landscape of tumor-infiltrating immune cell and immunomodulators in lung cancer. Biomed Pharmacother. 2017; 95:55-61.

https://doi.org/10.1016/i.biopha.2017.08.003

PMID:28826097

41. Bauer C, Kühnemuth B, Duewell P, Ormanns S, Gress T, Schnurr M. Prevailing over $T$ cell exhaustion: New developments in the immunotherapy of pancreatic cancer. Cancer Lett. 2016; 381:259-68.

https://doi.org/10.1016/i.canlet.2016.02.057 PMID:26968250

42. Yang K, Zhou J, Chen Y, Chen Y, Chen L, Zhang P, Ma L, Jiang Z, Bian J, Yin W. Angiotensin II contributes to intratumoral immunosuppressionvia induction of PDL1 expression in non-small cell lung carcinoma. Int Immunopharmacol. 2020; 84:106507. https://doi.org/10.1016/i.intimp.2020.106507 PMID:32339920

43. Dvinge $H$, Git $A$, Gräf $S$, Salmon-Divon $M$, Curtis $C$, Sottoriva A, Zhao $Y$, Hirst $M$, Armisen J, Miska EA, Chin SF, Provenzano E, Turashvili G, et al. The shaping and functional consequences of the microRNA landscape in breast cancer. Nature. 2013; 497:378-82.

https://doi.org/10.1038/nature12108

PMID:23644459

44. Hu Z, Chen X, Zhao Y, Tian T, Jin G, Shu Y, Chen Y, Xu L, Zen $K$, Zhang $C$, Shen $H$. Serum microRNA signatures identified in a genome-wide serum microRNA expression profiling predict survival of non-small-cell lung cancer. J Clin Oncol. 2010; 28:1721-6.

https://doi.org/10.1200/JCO.2009.24.9342

PMID:20194856

45. Li B, Cui Y, Diehn M, Li R. Development and Validation of an Individualized Immune Prognostic Signature in Early-Stage Nonsquamous Non-Small Cell Lung Cancer. JAMA Oncol. 2017; 3:1529-37.

https://doi.org/10.1001/jamaoncol.2017.1609 PMID:28687838

46. Chen $\mathrm{H}$, Carrot-Zhang J, Zhao $\mathrm{Y}, \mathrm{Hu} \mathrm{H}$, Freeman SS, Yu S, Ha G, Taylor AM, Berger AC, Westlake L, Zheng Y, Zhang J, Ramachandran A, et al. Genomic and immune profiling of pre-invasive lung adenocarcinoma. Nat Commun. 2019; 10:5472.

https://doi.org/10.1038/s41467-019-13460-3

PMID:31784532

47. Song Q, Shang J, Yang Z, Zhang L, Zhang C, Chen J, Wu $X$. Identification of an immune signature predicting prognosis risk of patients in lung adenocarcinoma. J Transl Med. 2019; 17:70.

https://doi.org/10.1186/s12967-019-1824-4

PMID:30832680

48. Wu GC, Pan HF, Leng RX, Wang DG, Li XP, Li XM, Ye DQ. Emerging role of long noncoding RNAs in autoimmune diseases. Autoimmun Rev. 2015; 14:798-805. https://doi.org/10.1016/i.autrev.2015.05.004 PMID:25989481

49. Heward JA, Lindsay MA. Long non-coding RNAs in the regulation of the immune response. Trends Immunol. 2014; 35:408-19.

https://doi.org/10.1016/i.it.2014.07.005 PMID:25113636

50. Xu M, Xu X, Pan B, Chen X, Lin K, Zeng K, Liu X, Xu T, Sun L, Qin J, He B, Pan Y, Sun H, Wang S. LncRNA SATB2-AS1 inhibits tumor metastasis and affects the tumor immune cell microenvironment in colorectal cancer by regulating SATB2. Mol Cancer. 2019; 18:135. https://doi.org/10.1186/s12943-019-1063-6 PMID:31492160

51. Rhee JK, Jung YC, Kim KR, Yoo J, Kim J, Lee YJ, Ko YH, Lee $\mathrm{HH}$, Cho BC, Kim TM. Impact of Tumor Purity on Immune Gene Expression and Clustering Analyses across Multiple Cancer Types. Cancer Immunol Res. 2018; 6:87-97.

https://doi.org/10.1158/2326-6066.CIR-17-0201 PMID:29141981

52. Yoshihara K, Shahmoradgoli M, Martínez E, Vegesna R, Kim H, Torres-Garcia W, Treviño V, Shen H, Laird PW, Levine DA, Carter SL, Getz G, Stemke-Hale K, et al. Inferring tumour purity and stromal and immune cell admixture from expression data. Nat Commun. 2013; $4: 2612$.

https://doi.org/10.1038/ncomms3612 PMID: 24113773

53. Yang J, Liang B, Hou S. TMPO-AS1 promotes cervical cancer progression by upregulating RAB14 via sponging miR-577. J Gene Med. 2019; 21:e3125. https://doi.org/10.1002/igm.3125 PMID:31483914

54. Mu X, Wu H, Liu J, Hu X, Wu H, Chen L, Liu W, Luo S, Zhao Y. Long noncoding RNA TMPO-AS1 promotes lung adenocarcinoma progression and is negatively regulated by miR-383-5p. Biomed Pharmacother. 2020; 125:109989.

https://doi.org/10.1016/j.biopha.2020.109989 PMID: $\underline{32062549}$ 


\section{SUPPLEMENTARY MATERIALS}

\section{Supplementary Files}

Please browse Full Text version to see the data of Supplementary Files 1, 2.

Supplementary File 1. The screened data in the immune system process M13664 of molecular signatures database.

Supplementary File 2. The screened data in the immune response M19817 of molecular signatures database. 